TRANSACTIONS OF THE

AMERICAN MATHEMATICAL SOCIETY

Volume 353, Number 11, Pages 4319-4332

S 0002-9947(01)02783-0

Article electronically published on June 14, 2001

\title{
UNIQUENESS OF VOLUME-MINIMIZING SUBMANIFOLDS CALIBRATED BY THE FIRST PONTRYAGIN FORM
}

\author{
DANIEL A. GROSSMAN AND WEIQING GU
}

\begin{abstract}
One way to understand the geometry of the real Grassmann manifold $G_{k}\left(\mathbf{R}^{k+n}\right)$ parameterizing oriented $k$-dimensional subspaces of $\mathbf{R}^{k+n}$ is to understand the volume-minimizing subvarieties in each homology class. Some of these subvarieties can be determined by using a calibration. In previous work, one of the authors calculated the set of 4-planes calibrated by the first Pontryagin form $p_{1}$ on $G_{k}\left(\mathbf{R}^{k+n}\right)$ for all $k, n \geq 4$, and identified a family of mutually congruent round 4 -spheres which are consequently homologically volume-minimizing. In the present work, we associate to the family of calibrated planes a Pfaffian system on the symmetry group $S O(k+n, \mathbf{R})$, an analysis of which yields a uniqueness result; namely, that any connected submanifold of $G_{k}\left(\mathbf{R}^{k+n}\right)$ calibrated by $p_{1}$ is contained in one of these 4 -spheres. A similar result holds for $p_{1}$-calibrated submanifolds of the quotient Grassmannian $G_{k}^{\natural}\left(\mathbf{R}^{k+n}\right)$ of non-oriented $k$-planes.
\end{abstract}

\section{INTRODUCTION}

1.1. Background. Due to the importance of the Grassmann manifolds parameterizing oriented subspaces of a vector space in both topology (where they support the universal characteristic classes) and geometry (where they provide a natural setting for innumerable interesting problems), most aspects of their own topology and geometry have been investigated and understood. However, one important feature that remains unknown is a complete list of closed subvarieties of Grassmannians which are volume-minimizing in their respective homology classes. In their fundamental work 8], Harvey and Lawson suggested the use of certain natural differential forms as calibrations in order to determine these subvarieties. The particular forms suggested are the invariant closed forms representing the universal characteristic classes for vector bundles (see [10], for instance).

This suggestion was first taken up in [4], with the use of Euler forms to determine which among the natural inclusions $G_{k^{\prime}}\left(\mathbf{R}^{k^{\prime}+n^{\prime}}\right) \subset G_{k}\left(\mathbf{R}^{k+n}\right)$ are homologically volume-minimizing. In the more ambitious work [3], volume-minimizing representative cycles were found for almost all homology classes in $G_{k}\left(\mathbf{R}^{k+n}\right)$, with $k, n \leq 4$, using as calibrations Euler forms, dual Euler forms, the first Pontryagin form $p_{1}$, and quaternionic forms. One of the present authors extended part of the preceding results in [7], proving that the 4 -cycles in $G_{4}\left(\mathbf{R}^{8}\right)$ calibrated by $p_{1}$

Received by the editors April 1, 2000 and, in revised form, September 23, 2000.

2000 Mathematics Subject Classification. Primary 53C38; Secondary 58A17, 53C40.

Key words and phrases. Calibrated geometry, Pontryagin form, Pfaffian systems.

The first author's research was supported by a fellowship from the Alfred P. Sloan foundation. 
remain calibrated by $p_{1}$ - and therefore homologically volume-minimizing - in all larger Grassmannians $G_{k}\left(\mathbf{R}^{k+n}\right) \supset G_{4}\left(\mathbf{R}^{8}\right)$.

The question of whether these volume-minimizing representatives are unique (up to congruence) naturally arises. For the Euler-calibrated sub-Grassmannians studied in 4], an affirmative answer was proven in the same paper. In his thesis [11, L.-H. Pan proved that the maximal connected submanifolds in $G_{4}\left(\mathbf{R}^{8}\right)$ calibrated by $p_{1}$ are indeed unique modulo ambient isometry using uniqueness theorems about sphere fibrations; however, it is known (see [3, p. 392]) that for certain other classes of $G_{4}\left(\mathbf{R}^{8}\right)$, the corresponding statement is false. In another thesis [9, C. Michael described submanifolds of $G_{3}\left(\mathbf{R}^{6}\right)$ calibrated by $p_{1}$ as corresponding to integral manifolds of a certain Pfaffian system on the Lie group $S O(6, \mathbf{R})$; an analysis of this system gave a proof that every connected $p_{1}$-calibrated submanifold in $G_{3}\left(\mathbf{R}^{6}\right)$ is congruent to part of the singular cycle PONT, defined in 3, p. 375]. The method is similar to that used by Robert Bryant (as reported in [3, pp. 378-380]) to prove non-existence of $p_{1}$-calibrated submanifolds in $G_{3}\left(\mathbf{R}^{7}\right)$.

In the present paper, we extend Michael's method to prove the following.

Theorem 1. Given any $p_{1}$-calibrated oriented 4-plane $V_{g} \subset T_{p} G_{k}\left(\mathbf{R}^{k+n}\right)$, with $k, n \geq 4$, there exists a unique maximal connected $p_{1}$-calibrated submanifold containing $p$ and tangent to $V_{g}$; any connected $p_{1}$-calibrated submanifold lies in a maximal one.

As explained below, a global uniqueness result follows from this.

Corollary 1. Any two maximal connected $p_{1}$-calibrated submanifolds of $G_{k}\left(\mathbf{R}^{k+n}\right)$ are congruent under the ambient isometry group.

The proof of Theorem 1 ends with a description of one maximal calibrated submanifold, which has the structure of a quaternionic projective line $\mathbf{H P}^{1}$, and is geometrically a round 4-sphere; this was already present in [3] and [7]. All of the results mentioned so far concern the Grassmannian of oriented subspaces of a real vector space; we also prove the same uniqueness theorem for the Grassmannian $G_{k}^{\natural}\left(\mathbf{R}^{k+n}\right)$ of non-oriented subspaces of a real vector space.

The difficulty of using calibrations to study homologically volume-minimizing submanifolds often lies in determining the calibrated planes, those subspaces of the tangent space on which the closed differential form being used as a calibration takes its maximum value; this value is called the comass of the calibration. This formidable computation was carried out for the first Pontryagin form $p_{1}$ in [3] for low-dimensional cases, and the result was used in 9] and 11] to obtain the low-dimensional uniqueness theorems. In [7, by introducing a new quaternionic symmetrization scheme, one of the present authors was able to systematically calculate the comasses and calibrated planes of $p_{1}$ on all real Grassmannians; recently, Dadok and Harvey (2]) have given a simplified version of this calculation. Gu's main result is that each of the $p_{1}$-calibrated 4-planes of $G_{k}\left(\mathbf{R}^{k+n}\right)$ (with $k, n \geq 4$ ) can be carried by an isometry to one of the previously known $p_{1}$-calibrated 4-planes of a sub-Grassmannian $G_{4}\left(\mathbf{R}^{8}\right) \subset G_{k}\left(\mathbf{R}^{k+n}\right)$; the isometry group of $G_{4}\left(\mathbf{R}^{8}\right)$ was already known to act transitively on the latter set. This "infinitesimal uniqueness theorem", combined with Pan's $p_{1}$-uniqueness result in [11] on $G_{4}\left(\mathbf{R}^{8}\right)$, makes plausible the general uniqueness result expressed in Corollary 1 (although we do not actually use Pan's result). Note that Gu's proof of transitivity of the isometry group acting on calibrated 4-planes, combined with Theorem 1 implies Corollary 1 
One way of interpreting this result is as the simplifying observation that the 4dimensional, $p_{1}$-calibrated geometry of the real Grassmannian $G_{k}\left(\mathbf{R}^{k+n}\right)$ is already fully present in the sub-Grassmannian $G_{4}\left(\mathbf{R}^{8}\right)$. The $p_{1}$-calibrated oddities in low dimensions - the singular cycle PONT in $G_{3}\left(\mathbf{R}^{6}\right)$, and the non-existence in $G_{3}\left(\mathbf{R}^{7}\right)$, for example-do not reappear.

1.2. Overview. The layout of this paper is as follows.

In Section 2, we first review the basic notions of calibrated geometry; for much more, see [8]. We also introduce the Grassmannian of oriented subspaces of a real vector space as a homogeneous space of the group $S O(k+n, \mathbf{R})$, and we use the infinitesimal structure of the group to define both a Riemannian metric and a closed differential form - the first Pontryagin form $p_{1}$ - on the Grassmannian. We then introduce a larger symmetry group $O^{\varepsilon}(k+n)$ of the Grassmannian, which has the advantage of acting transitively on the $p_{1}$-calibrated planes. These planes are described again through the use of the infinitesimal group structure, as expressed in the left-invariant Maurer-Cartan 1-form and its exterior derivative.

In Section 3, we compute the infinitesimal stabilizer of the action of the symmetry group $O^{\varepsilon}(k+n)$ on the set of $p_{1}$-calibrated planes of the Grassmannian. This information complements our description of the calibrated planes given in the preceding section to give a more complete picture of the local differential geometry, as expressed on the symmetry group.

Section 4 is the heart of the paper, in which we prove Theorem 11 The statement echoes the conclusion of the Frobenius theorem about foliations of manifolds, because the latter theorem carries the essential integrability results needed to give a complete local description of our calibrated submanifolds. The bulk of the proof involves constructing a Pfaffian system, or subbundle of a cotangent bundle, which satisfies the hypotheses of the Frobenius theorem and whose integral manifolds correspond to $p_{1}$-calibrated submanifolds of the Grassmannian. This Pfaffian system is defined on the symmetry group $O^{\varepsilon}(k+n)$, where computations are much easier because of the invariant expressions for all relevant quantities. We actually begin with a Pfaffian system not satisfying the hypotheses of the Frobenius theorem; subjecting this system to two partial prolongations gives the desired system, and an interpretation of this final system completes the proof.

Finally, in Section 5 , the analogous results are stated and justified for the Grassmannian of non-oriented subspaces of a real vector space.

Some remaining areas of investigation are the following.

First, for the low-dimensional real Grassmannians, various invariant forms were used as calibrations in 3] to compute calibrated cycles in almost every homology class. What kind of uniqueness results hold for these other calibrations, among which are Euler forms, dual Euler forms, and the dual of the first Pontryagin form, in higher-dimensional real Grassmannians?

Second, to our knowledge, nothing is known about either planes or submanifolds of the real Grassmannians calibrated by the higher Pontryagin forms. For instance, is all of the $p_{2}$-calibrated geometry of $G_{k}\left(\mathbf{R}^{k+n}\right)$ (for $k, n \geq 8$ ) already present in $G_{8}\left(\mathbf{R}^{16}\right)$ ?

Third, a topic of current investigation by the authors ([6]) is the 4-dimensional geometry of complex Grassmannians calibrated by the second Chern form $c_{2}$. The main difficulty with this and the previously suggested problems is, as usual, determination of the calibrated planes. 
1.3. Notation. Throughout this paper, we use the following conventions.

The space $\mathbf{R}^{4 \times 4}$ of matrices is identified with the space $\operatorname{End}_{\mathbf{R}}(\mathbf{H})$ of real-linear endomorphisms of the quaternions, described with respect to the standard basis $\{\mathbf{1}, \mathbf{i}, \mathbf{j}, \mathbf{k}\}$. More explicitly, we will refer to

$$
\begin{aligned}
R_{\mathbf{i}} & =\left(\begin{array}{cccc}
0 & -1 & 0 & 0 \\
1 & 0 & 0 & 0 \\
0 & 0 & 0 & 1 \\
0 & 0 & -1 & 0
\end{array}\right), R_{\mathbf{j}}=\left(\begin{array}{cccc}
0 & 0 & -1 & 0 \\
0 & 0 & 0 & -1 \\
1 & 0 & 0 & 0 \\
0 & 1 & 0 & 0
\end{array}\right), R_{\mathbf{k}}=\left(\begin{array}{cccc}
0 & 0 & 0 & -1 \\
0 & 0 & 1 & 0 \\
0 & -1 & 0 & 0 \\
1 & 0 & 0 & 0
\end{array}\right), \\
L_{\mathbf{i}} & =\left(\begin{array}{cccc}
0 & -1 & 0 & 0 \\
1 & 0 & 0 & 0 \\
0 & 0 & 0 & -1 \\
0 & 0 & 1 & 0
\end{array}\right), L_{\mathbf{j}}=\left(\begin{array}{cccc}
0 & 0 & -1 & 0 \\
0 & 0 & 0 & 1 \\
1 & 0 & 0 & 0 \\
0 & -1 & 0 & 0
\end{array}\right), L_{\mathbf{k}}=\left(\begin{array}{cccc}
0 & 0 & 0 & -1 \\
0 & 0 & -1 & 0 \\
0 & 1 & 0 & 0 \\
1 & 0 & 0 & 0
\end{array}\right) .
\end{aligned}
$$

These give a basis for the subspace $\mathfrak{s o}(4, \mathbf{R}) \subset \mathbf{R}^{4 \times 4}$ of skew-symmetric matrices. In fact,

$$
\mathbf{R}^{4 \times 4}=(\mathbf{R} \cdot I) \oplus\left(\left(\bigoplus_{\mu} \mathbf{R} \cdot L_{\mu}\right) \oplus\left(\bigoplus_{\nu} \mathbf{R} \cdot R_{\nu}\right)\right) \oplus\left(\bigoplus_{\mu, \nu} \mathbf{R} \cdot\left(L_{\mu} R_{\nu}\right)\right),
$$

where $\mu, \nu \in\{\mathbf{i}, \mathbf{j}, \mathbf{k}\}$ throughout. This gives the usual decomposition of square matrices into pure-trace, skew-symmetric, and traceless-symmetric parts, respectively, with the second summand further decomposed into two subalgebras isomorphic to $\mathfrak{s p}(1) \cong \mathfrak{s u}(2)$, as is characteristic of 4 -dimensional geometry. By construction, multiplication of the matrices corresponds to composition of operators on $\mathbf{H}$, simplifying some otherwise-tedious calculations.

Always, we have $k, n \geq 4$. In this generality, we will take advantage of (11) by using the decomposition

$$
\mathbf{R}^{k+n} \cong \mathbf{H} \oplus \mathbf{R}^{k-4} \oplus \mathbf{H}^{\prime} \oplus \mathbf{R}^{n-4},
$$

where $\mathbf{H}^{\prime}$ is simply another copy of the quaternions. We will also use a standard inclusion $G_{4}\left(\mathbf{R}^{8}\right) \hookrightarrow G_{k}\left(\mathbf{R}^{k+n}\right)$ defined by identifying $E^{4} \subset \mathbf{R}^{8} \cong \mathbf{H} \oplus \mathbf{H}^{\prime}$ with $E \oplus \mathbf{R}^{k-4} \subset \mathbf{R}^{k+n}$, referring to the terms of (2).

Finally, we will often use the same notation for both a Pfaffian system, regarded as a vector subbundle of a cotangent bundle, and for the exterior ideal algebraically generated by that subbundle.

Acknowledgements. The authors met at MSRI in July 1999, at a summer program on exterior differential systems. Stimulating lectures by Robert Bryant and Jeanne Clelland, as well as several helpful conversations with each of them, provided some of the techniques used in this work. We thank them for this, and we thank MSRI for sponsoring the program from which we learned so much.

\section{4-Planes Calibrated By $p_{1}$}

2.1. Calibrations. A calibration is a closed differential $p$-form $\psi$ on an oriented Riemannian manifold $\mathcal{M}$, whose values on the set of decomposable unit $p$-vectors (representing oriented $p$-dimensional tangent subspaces) are bounded. The supremum of these values, taken over all $p$-planes at all points of the manifold, is called 
the comass of $\psi$, denoted by $\|\psi\|^{*}$. An oriented tangent $p$-plane is said to be calibrated by $\psi$, or $\psi$-calibrated, if

$$
\left\langle\psi, v_{1} \wedge \cdots \wedge v_{p}\right\rangle=\|\psi\|^{*}
$$

for some (and thus any) oriented, orthonormal basis $v_{i}$. An oriented $p$-dimensional submanifold $S \subset \mathcal{M}$ is said to be $\psi$-calibrated if each of its tangent spaces is a $\psi$-calibrated plane. In case $S$ is a closed, $\psi$-calibrated submanifold representing a homology class $[S] \in H_{p}(\mathcal{M}, \mathbf{R})$, and $S^{\prime} \sim S$ represents the same class, the fundamental calculation is

$$
\|\psi\|^{*} \cdot \operatorname{Vol}(S)=\int_{S} \psi=\int_{S^{\prime}} \psi \leq\|\psi\|^{*} \cdot \operatorname{Vol}\left(S^{\prime}\right),
$$

proving that $S$ is volume-minimizing in its homology class, with equality holding if and only if $S^{\prime}$ is also $\psi$-calibrated.

This is the basic method of calibration by which one proves certain submanifolds homologically volume-minimizing. It will be applied to the Grassmannian of oriented subspaces of a real vector space, with the first Pontryagin form as a calibration. Typically, a major difficulty is in determining the calibrated planes; in our case, this has been done in [7.

It is worth noting that in recent years the notion of calibration has found other application, most notably in M. Gromov's work 5] on sub-Riemannian geometry, in Gang Tian's work 12] on higher-dimensional gauge theory, and in various works (see 1], for example) of D. DeTurck et al. on isospectral deformations of Riemannian manifolds.

2.2. The Structure of the Real, Oriented Grassmannian. The manifold $G_{k}\left(\mathbf{R}^{k+n}\right)$ parameterizing oriented $k$-dimensional subspaces in real Euclidean space $\mathbf{R}^{k+n}$ acquires the structure of a homogeneous space by the fibration

$$
\pi: S O(k+n, \mathbf{R}) \rightarrow G_{k}\left(\mathbf{R}^{k+n}\right), \quad\left(f_{1} \cdots f_{k+n}\right) \mapsto\left[f_{1} \wedge \cdots \wedge f_{k}\right],
$$

where $f_{i}$ are columns, considered as elements of $\mathbf{R}^{k+n}$, and the notation on the right is the usual way of denoting an oriented subspace in terms of a basis. The isotropy subgroup of the familiar left-action (that is, the stabilizer of the image $\pi(e)$ of the identity $e \in S O(k+n, \mathbf{R}))$ is equal to the block-diagonal $S O(k, \mathbf{R}) \times$ $S O(n, \mathbf{R}) \subset S O(k+n, \mathbf{R})$, which is therefore the structure group of $\pi$ as a rightprincipal bundle. The inner-product $(x, y) \mapsto \operatorname{Tr}\left(x^{t} y\right)$ on $T_{e} S O(k+n, \mathbf{R}) \cong$ $\mathfrak{s o}(k+n, \mathbf{R})$ is invariant under the adjoint action of $S O(k, \mathbf{R}) \times S O(n, \mathbf{R})$ on $\mathfrak{s o}(k+n, \mathbf{R})$, and therefore defines a Riemannian metric on the quotient $G_{k}\left(\mathbf{R}^{k+n}\right)$. The first Pontryagin form $p_{1}$ is a closed, $S O(k+n, \mathbf{R})$-invariant differential 4 -form, representing in de Rham cohomology $H^{4}\left(G_{k}\left(\mathbf{R}^{k+n}\right), \mathbf{R}\right)$ the universal characteristic class of the same name; we will define it precisely below. This $p_{1}$ calibrates certain oriented 4-planes in tangent spaces $T_{x} G_{k}\left(\mathbf{R}^{k+n}\right)$. These $p_{1}$-calibrated planes were found and described in [3] for the case $k=n=4$; in [7] (and again in [2]), the description was extended to arbitrary $k, n \geq 4$, and our goal in this section is to describe them in a manner suited to our further analysis.

The left-invariant, $\mathfrak{s o}(k+n, \mathbf{R})$-valued Maurer-Cartan form $\varphi$ on $S O(k+n, \mathbf{R})$ gives a linear isomorphism from each tangent space $T_{g} S O(k+n, \mathbf{R})$ to the Lie algebra $\mathfrak{s o}(k+n, \mathbf{R})$; it may be written as

$$
\varphi=\left(\begin{array}{cc}
\alpha & -{ }^{t} \omega \\
\omega & \beta
\end{array}\right)
$$


where $\alpha=-{ }^{t} \alpha$ is a $k \times k$ matrix of one-forms, $\omega$ is an $n \times k$ matrix of one-forms, and $\beta=-{ }^{t} \beta$ is an $n \times n$ matrix of one-forms. It is easy to verify that at each $g \in S O(k+n, \mathbf{R})$, the block $\omega$ induces a linear isomorphism

$$
\omega_{g}: T_{\pi(g)} G_{k}\left(\mathbf{R}^{k+n}\right) \rightarrow \mathbf{R}^{n \times k}
$$

However, different $g \neq g^{\prime}$ with $\pi(g)=\pi\left(g^{\prime}\right)$ may induce different identifications $\omega_{g} \neq \omega_{g^{\prime}}: T_{\pi(g)} G_{k}\left(\mathbf{R}^{k+n}\right) \rightarrow \mathbf{R}^{n \times k}$. In fact, for $g^{\prime}=g h$ with $h \in S O(k, \mathbf{R}) \times$ $S O(n, \mathbf{R})$ we have the equivariance

$$
\omega_{g^{\prime}}=\omega_{g h}=B^{-1} \omega_{g} A, \quad \text { for } h=\left(\begin{array}{cc}
A & 0 \\
0 & B
\end{array}\right) .
$$

Now, the differential 4-form on $S O(k+n, \mathbf{R})$ given by

$$
\text { Trace }\left(\omega \wedge{ }^{t} \omega \wedge \omega \wedge{ }^{t} \omega\right)
$$

is at each point the pull-back under $\pi^{*}$ of some form on $G_{k}\left(\mathbf{R}^{k+n}\right)$; and from (4) one can see that it is invariant under fiber-translation by $h \in S O(k, \mathbf{R}) \times S O(n, \mathbf{R})$. This implies that it is the pull-back of some unique 4 -form $p_{1}$ on $G_{k}\left(\mathbf{R}^{k+n}\right)$, which is by definition the first Pontryagin form.

2.3. The $p_{1}$-Calibrated Planes. The set of $p_{1}$-calibrated 4-planes in $T_{x} G_{k}\left(\mathbf{R}^{k+n}\right)$ includes the oriented 4-planes corresponding-via $\omega_{g}$ for some $g \in \pi^{-1}(x) \subset$ $S O(k+n, \mathbf{R})$ - to the following oriented subspace of $\mathbf{R}^{n \times k}$ :

$$
\begin{gathered}
V=\left\{\left(\begin{array}{cc}
\bar{v} & \mathbf{0}_{4 \times(k-4)} \\
\mathbf{0}_{(n-4) \times 4} & \mathbf{0}_{(n-4) \times(k-4)}
\end{array}\right): \bar{v} \in \operatorname{Span}\left\{I, R_{\mathbf{i}}, R_{\mathbf{j}}, R_{\mathbf{k}}\right\} \subset \mathbf{R}^{4 \times 4}\right\}, \\
\text { oriented with } R_{\mathbf{i}} \wedge R_{\mathbf{j}} \wedge R_{\mathbf{k}} \wedge I>0 .
\end{gathered}
$$

(We have taken some liberty in identifying $R_{\nu}$ with an $n \times k$ matrix in an obvious way.)

However, there are more calibrated planes than these; the space described above is only one of four connected components of the space of all calibrated oriented 4 -planes. The others are images of the described component under the group generated by a commuting pair of order-2 isometries of $G_{k}\left(\mathbf{R}^{k+n}\right)$, each of which preserves $p_{1}$, and which we shall now describe (see [3. p. 393] and [13, pp. 302-315] for more details).

For the first, we can take the motion induced by any orientation-reversing orthogonal involution on $\mathbf{R}^{k+n}$, i.e., any element of $O(k+n, \mathbf{R}) \backslash S O(k+n, \mathbf{R})$ of order 2 . For instance, reversal of one of the coordinate axes will suffice. This simply enlarges our symmetry group to $O(k+n, \mathbf{R})$, and our isotropy group to $S O(k, \mathbf{R}) \times O(n, \mathbf{R})$; we could have begun by defining the fibration $\pi$ on $O(k+n, \mathbf{R})$, but find it preferable to highlight the presence of the extra components.

The second isometry $\varepsilon$ takes an oriented $k$-subspace in $\mathbf{R}^{k+n}$ to that same subspace, but with the opposite orientation.

The group of motions of $G_{k}\left(\mathbf{R}^{k+n}\right)$ generated by $\varepsilon$ and the induced action of $O(k+n, \mathbf{R})$ is the full simultaneous symmetry group of both the invariant metric and the closed form $p_{1}$. Consequently, the entire group must act on the space of calibrated planes; it is not difficult to verify that the action is effective, so we really could not have used any smaller group. We denote the group by $O^{\varepsilon}(k+n)$, and remind the reader that its identity component is isomorphic to $S O(k+n, \mathbf{R})$. Therefore, all that we have said about the $\mathfrak{s o}(k+n, \mathbf{R})$-valued Maurer-Cartan 
form $\varphi$ applies to $O^{\varepsilon}(k+n)$, including the formula for the pull-back of $p_{1}$; the identification of tangent spaces of $G_{k}\left(\mathbf{R}^{k+n}\right)$ with $\mathbf{R}^{n \times k}$ by $\omega$; and the description of $p_{1}$-calibrated planes as corresponding via $\omega_{g}$ to the oriented 4 -space $V \subset \mathbf{R}^{n \times k}$ except that, by construction, all of the $p_{1}$-calibrated planes occur in the latter manner for $g \in O^{\varepsilon}(k+n)$. Also, the reader can verify that the isotropy group of $O^{\varepsilon}(k+n)$ acting on $G_{k}\left(\mathbf{R}^{k+n}\right)$ is isomorphic to $O(k, \mathbf{R}) \times O(n, \mathbf{R})$, and that the same equivariance (4) holds, with $A \in O(k, \mathbf{R}), B \in O(n, \mathbf{R})$ (in which the four components are now apparent).

In fact, it is on $O^{\varepsilon}(k+n)$ that our computations in the proof of Theorem 1 will take place; but because these computations are inherently local, the reader need only be comfortable with $\mathfrak{s o}(k+n, \mathbf{R})$ to understand them.

In the next section we will determine the Lie algebra of the stabilizer of the particular calibrated oriented 4-plane $\omega_{e}^{-1}(V) \subset T_{\pi(e)} G_{k}\left(\mathbf{R}^{k+n}\right)$. This will help us to better understand the space of calibrated planes, to set up the Pfaffian system describing $p_{1}$-calibrated submanifolds of $G_{k}\left(\mathbf{R}^{k+n}\right)$, and to interpret the results of the analysis of that system.

\section{Infinitesimal Stabilizer of a $p_{1}$-Calibrated Plane}

In this section, we calculate the Lie algebra of the stabilizer in $O(k, \mathbf{R}) \times O(n, \mathbf{R})$ of a particular $p_{1}$-calibrated oriented 4-plane $V_{e} \subset T_{\pi(e)} G_{k}\left(\mathbf{R}^{k+n}\right)$. Knowledge of this "infinitesimal stabilizer" will be useful later for guiding us through the analysis of our Pfaffian system. The map $\omega_{e}: T_{\pi(e)} G_{k}\left(\mathbf{R}^{k+n}\right) \stackrel{\sim}{\longrightarrow} \mathbf{R}^{n \times k}$ conjugates the isotropy action of $O(k, \mathbf{R}) \times O(n, \mathbf{R})$ on $T_{\pi(e)} G_{k}\left(\mathbf{R}^{k+n}\right)$ into a left-action on $\mathbf{R}^{n \times k}$ given by

$$
(A, B) \cdot v=B v A^{-1} .
$$

We are therefore interested in understanding the stabilizer under this action of the oriented subspace $V=\omega\left(V_{e}\right) \subset \mathbf{R}^{n \times k}$ (see (5)) corresponding to the calibrated plane $V_{e}$. We will first consider the case $k=n=4$, the result of which will then be useful in the general case $k, n \geq 4$.

In case $k=n=4$, recall that $V=\operatorname{Span}\left\{I, R_{\mathbf{i}}, R_{\mathbf{j}}, R_{\mathbf{k}}\right\} \subset \mathbf{R}^{4 \times 4}$. The condition that $(a, b) \in \mathfrak{s o}(4, \mathbf{R}) \times \mathfrak{s o}(4, \mathbf{R})$ be in the Lie algebra of the stabilizer of $V$ is that

$$
b v-v a \in V \quad \text { for all } v=v^{1} I+v^{\nu} R_{\nu} \in V,
$$

where summation over $\nu \in\{\mathbf{i}, \mathbf{j}, \mathbf{k}\}$ is understood throughout. For $a, b \in \mathfrak{s o}(4, \mathbf{R})$ given by $a=a_{L}^{\mu} L_{\mu}+a_{R}^{\nu} R_{\nu}, \quad b=b_{L}^{\mu} L_{\mu}+b_{R}^{\nu} R_{\nu}$ (where $a_{L}^{\mu}, a_{R}^{\nu}, b_{L}^{\mu}, b_{R}^{\nu} \in \mathbf{R}$ ), this condition immediately gives

$$
\begin{aligned}
b_{L}^{\mu} v^{1}-v^{1} a_{L}^{\mu}=0 & \text { for all } v^{1} \in \mathbf{R}, \mu \in\{\mathbf{i}, \mathbf{j}, \mathbf{k}\}, \text { and } \\
b_{L}^{\mu} v^{\nu}-v^{\nu} a_{L}^{\mu}=0 & \text { for all } v^{\nu} \in \mathbf{R}, \mu, \nu \in\{\mathbf{i}, \mathbf{j}, \mathbf{k}\},
\end{aligned}
$$

from which we obtain the condition $a_{L}^{\mu}=b_{L}^{\mu}$; in other words, the "left"-components of $a, b \in \mathfrak{s o}(4, \mathbf{R}) \cong \mathfrak{s p}(1)_{L} \times \mathfrak{s p}(1)_{R}$ must be equal (see (1)), while the "right"components are arbitrary. It is now clear that the infinitesimal stabilizer is isomorphic to $\mathfrak{s p}(1)^{3} \cong \mathfrak{s p}(1) \times \mathfrak{s o}(4, \mathbf{R})$.

In the general case $k, n \geq 4$, the condition that $(a, b) \in \mathfrak{s o}(k, \mathbf{R}) \times \mathfrak{s o}(n, \mathbf{R})$ be in the Lie algebra of the stabilizer of $V$ defined in (5) is that

$$
b v-v a \in V \quad \text { for all } v=\left(\begin{array}{cc}
\bar{v} & 0 \\
0 & 0
\end{array}\right), \bar{v}=v^{1} I+v^{\nu} R_{\nu} .
$$


When we use block notation

$$
a=\left(\begin{array}{cc}
a_{1} & -{ }^{t} a_{2} \\
a_{2} & a_{4}
\end{array}\right), \quad b=\left(\begin{array}{cc}
b_{1} & -{ }^{t} b_{2} \\
b_{2} & b_{4}
\end{array}\right),
$$

with $a_{1} \in \mathfrak{s o}(4, \mathbf{R}), a_{4} \in \mathfrak{s o}(k-4, \mathbf{R}), a_{2} \in \mathbf{R}^{(k-4) \times 4}, \quad b_{1} \in \mathfrak{s o}(4, \mathbf{R}), \quad b_{4} \in$ $\mathfrak{s o}(n-4, \mathbf{R})$, and $b_{2} \in \mathbf{R}^{(n-4) \times 4}$, this condition gives

$$
\bar{v}^{t} a_{2}=0, \quad b_{2} \bar{v}=0, \quad b_{1} \bar{v}-\bar{v} a_{1} \in \operatorname{Span}\left\{I, R_{\mathbf{i}}, R_{\mathbf{j}}, R_{\mathbf{k}}\right\} .
$$

The first two of these equations imply that $a_{2}=b_{2}=0$, and the third describes the situation of the preceding case, $k=n=4$; clearly, $a_{4} \in \mathfrak{s o}(k-4, \mathbf{R})$ and $b_{4} \in \mathfrak{s o}(n-4, \mathbf{R})$ remain free. Therefore, we have proven the following.

Lemma 1. The subgroup of $O(k, \mathbf{R}) \times O(n, \mathbf{R})$ stabilizing the $p_{1}$-calibrated plane $V_{e}$ has Lie algebra isomorphic to $\mathfrak{s p}(1) \times \mathfrak{s o}(4, \mathbf{R}) \times \mathfrak{s o}(k-4, \mathbf{R}) \times \mathfrak{s o}(n-4, \mathbf{R})$, defined in the block form (6) by $a_{2}=0, b_{2}=0$, and referring to the decomposition (11), $a_{1 L}=b_{1 L}$.

The set of calibrated 4-planes over a single point $p \in G_{k}\left(\mathbf{R}^{k+n}\right)$ is a homogeneous space of $O^{\varepsilon}(k+n)$, and the reader should think of Lemma 1 as describing its local structure.

\section{Proof of Theorem 1}

4.1. Construction of the Associated Pfaffian System. Our interest is in describing the 4-dimensional oriented submanifolds $S \subset G_{k}\left(\mathbf{R}^{k+n}\right)$ all of whose tangent spaces are $p_{1}$-calibrated planes. To avoid confusion, in this section we will let $\mathcal{M}=G_{k}\left(\mathbf{R}^{k+n}\right)$. The set of all $p_{1}$-calibrated planes is a subbundle $G^{c a l} \rightarrow \mathcal{M}$ of the Grassmann bundle $G_{4}(T \mathcal{N}) \rightarrow \mathcal{M}$ of all oriented tangent 4-planes of $\mathcal{M}$; the fiber of the subbundle is the 4-component homogeneous space of $p_{1}$-calibrated planes at a point, locally described in the preceding section. The reader is advised to keep in mind our interpretation of the theorem as indicating that the $p_{1}$-calibrated geometry of $G_{k}\left(\mathbf{R}^{k+n}\right)$ is already completely expressed in the submanifold $G_{4}\left(\mathbf{R}^{8}\right) \subset G_{k}\left(\mathbf{R}^{k+n}\right)$.

Given any 4-dimensional oriented submanifold $S \subset \mathcal{M}$, we may lift it to the image of its Gauss map $\gamma_{S}: S \rightarrow G_{4}(T \mathcal{N})$ given by $s \mapsto T_{s} S \subset T_{s} \mathcal{M}$, where $T_{s} S$ denotes the tangent space of $S$. Clearly, $S \subset \mathcal{M}$ is calibrated by $p_{1}$ if and only if the image $\gamma_{S}(S)$ lies in the subset $G^{c a l} \subset G_{4}(T \mathcal{M})$. Of course, not every 4-dimensional submanifold in $G^{\text {cal }}$ arises this way, but we need not concern ourselves with the criterion (typically called a "contact condition") for detecting those that do.

Notice that the fibration $\pi: O^{\varepsilon}(k+n) \rightarrow \mathcal{M}$ lifts to give another fibration $\hat{\pi}: O^{\varepsilon}(k+n) \rightarrow G^{c a l}$, defined using (3) by $\hat{\pi}(g)=\omega_{g}^{-1}(V)$ :

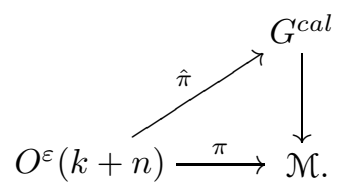

The isotropy subgroup of this lifted fibration $\hat{\pi}$ is the stabilizer of $V$ whose Lie algebra was described in Lemma 1, Given a calibrated submanifold $S \subset \mathcal{M}$, we are first going to consider its Gauss-image $\gamma_{S}(S) \subset G^{c a l}$, and then pull back to consider $\hat{S}=\hat{\pi}^{-1}\left(\gamma_{S}(S)\right) \subset O^{\varepsilon}(k+n)$. The motivation for the latter step is that we have a left-invariant basis for the differential 1-forms on the Lie group $O^{\varepsilon}(k+n)$ 
coming from the Maurer-Cartan form, and formulae for the derivatives of these 1-forms are available in the Maurer-Cartan equation (see (7) below). Note that $\hat{S}$ has dimension equal to $\operatorname{dim}(S)+\operatorname{dim}(\hat{\pi}$-fiber $)=4+3 \cdot 3+\left(\begin{array}{c}k-4 \\ 2\end{array}\right)+\left(\begin{array}{c}n-4 \\ 2\end{array}\right)$.

To prepare the computational apparatus, recall that we introduced in block form (of sizes $k, n$ ) the Maurer-Cartan form

$$
\varphi=\left(\begin{array}{cc}
\alpha & -{ }^{t} \omega \\
\omega & \beta
\end{array}\right) .
$$

We need to dissect it further, by writing

$$
\alpha=\left(\begin{array}{cc}
\alpha_{1} & -{ }^{t} \alpha_{2} \\
\alpha_{2} & \alpha_{4}
\end{array}\right), \quad \beta=\left(\begin{array}{cc}
\beta_{1} & -{ }^{t} \beta_{2} \\
\beta_{2} & \beta_{4}
\end{array}\right), \quad \omega=\left(\begin{array}{ll}
\omega_{1} & \omega_{3} \\
\omega_{2} & \omega_{4}
\end{array}\right),
$$

where the various smaller matrices satisfy

$$
\alpha_{1}, \beta_{1}, \omega_{1} \in \mathfrak{s o}(4, \mathbf{R}) \otimes \Omega^{1}, \quad \alpha_{4} \in \mathfrak{s o}(k-4, \mathbf{R}) \otimes \Omega^{1}, \quad \beta_{4} \in \mathfrak{s o}(n-4, \mathbf{R}) \otimes \Omega^{1},
$$

and the rest of the sizes are determined by these (with no further relations) 1 The skew-symmetric $4 \times 4$ matrices $\alpha_{1}, \beta_{1}, \omega_{1}$ will have their 1 -form components distinguished not by the usual "matrix-entry-index" notation but according to the invariant decomposition (1); namely,

$$
\begin{gathered}
\alpha_{1}=\alpha_{L}+\alpha_{R}=\alpha_{L}^{\mu} L_{\mu}+\alpha_{R}^{\nu} R_{\nu}, \quad \beta_{1}=\beta_{L}+\beta_{R}=\beta_{L}^{\mu} L_{\mu}+\beta_{R}^{\nu} R_{\nu}, \\
\omega_{1}=\omega_{I}+\left(\omega_{L}+\omega_{R}\right)+\omega_{L R}=\left(\omega^{1} I\right)+\left(\omega_{L}^{\mu} L_{\mu}+\omega_{R}^{\nu} R_{\nu}\right)+\left(\omega^{\mu \nu} L_{\mu} R_{\nu}\right),
\end{gathered}
$$

where $\alpha_{L}$, \&c., are $\mathbf{R}^{4 \times 4}$-valued 1-forms, with components $\alpha_{L}^{\mu}(\mu \in\{\mathbf{i}, \mathbf{j}, \mathbf{k}\}), \& c$., which are ordinary scalar-valued 1 -forms. It will be helpful sometimes to write

$$
\varphi=\left(\begin{array}{cccc}
\alpha_{1} & -{ }^{t} \alpha_{2} & -{ }^{t} \omega_{1} & -{ }^{t} \omega_{2} \\
\alpha_{2} & \alpha_{4} & -{ }^{t} \omega_{3} & -{ }^{t} \omega_{4} \\
\omega_{1} & \omega_{3} & \beta_{1} & -{ }^{t} \beta_{2} \\
\omega_{2} & \omega_{4} & \beta_{2} & \beta_{4}
\end{array}\right) .
$$

In case $k=4$ or $n=4$, some of these forms are absent; this renders some of the computations below vacuous, but no false statements follow.

The various identifications and maps described up to this point have been designed so that the following holds.

Lemma 2. Locally, $p_{1}$-calibrated manifolds $S \subset \mathcal{M}$ are in one-to-one correspondence (via $S \leftrightarrow \hat{S}$ ) with submanifolds of $O^{\varepsilon}(k+n)$ on which

$$
\omega_{L}=0, \omega_{L R}=0, \omega_{2}=0, \omega_{3}=0, \omega_{4}=0,
$$

and

$$
\omega^{1}, \omega_{R}^{\nu}, \alpha_{L}^{\mu}+\beta_{L}^{\mu}, \alpha_{R}^{\nu}, \beta_{R}^{\nu},\left(\alpha_{4}\right)_{j}^{i},\left(\beta_{4}\right)_{j}^{i}
$$

are everywhere linearly independent.

Here $\left(\alpha_{4}\right)_{j}^{i}$ denotes the collection of independent entries of the skew-symmetric matrix $\alpha_{4}$ (say, $1 \leq i<j \leq k-4$ ), and likewise for $\left(\beta_{4}\right)_{j}^{i}($ with $1 \leq i<j \leq n-4)$.

\footnotetext{
1 " $W \otimes \Omega^{1} "$ denotes the space of 1 -forms with values in the vector space $W$.
} 
Proof. If a point $g \in O^{\varepsilon}(k+n)$ lies in the lift $\hat{S}$ of a $p_{1}$-calibrated submanifold, then $\hat{S}$ must contain the fiber of $\hat{\pi}$ passing through $g$, on which (by Lemma 1) the 1 -forms $\alpha_{L}^{\mu}+\beta_{L}^{\mu}, \alpha_{R}^{\nu}, \beta_{R}^{\nu},\left(\alpha_{4}\right)_{j}^{i},\left(\beta_{4}\right)_{j}^{i}$ constitute a basis for all 1-forms. Furthermore, the tangent space of $S=\pi(\hat{S})$ at $\pi(g)$ must equal the calibrated 4-plane denoted by $\hat{\pi}(g)$, by construction of the lift $\hat{S}$; consequently, $\omega_{g}$ restricted to $\hat{S}$ takes values only in the space $V$ of (5), which is to say that

$$
\omega_{L}=0, \omega_{L R}=0, \omega_{2}=0, \omega_{3}=0, \text { and } \omega_{4}=0 .
$$

Finally, because $S$ has dimension 4 , and the various components of $\omega$ must span the cotangent space of any local section of $\hat{S} \rightarrow S$ (which is transverse to the $\hat{\pi}$-fibers previously considered), it is necessary that $\omega^{1}$ and $\omega_{R}^{\nu}$ be independent from each other and from $\alpha_{L}^{\mu}+\beta_{L}^{\mu}, \alpha_{R}^{\nu}, \beta_{R}^{\nu},\left(\alpha_{4}\right)_{j}^{i}$, and $\left(\beta_{4}\right)_{j}^{i}$. From these observations, our statement of Lemma 2 follows easily.

4.2. Analysis of the Pfaffian System. We now have a description of the $p_{1}$ calibrated manifolds in terms of a Pfaffian system with independence condition on the Lie group $O^{\varepsilon}(k+n)$. We will see that this Pfaffian system is not integrable; that is, it does not satisfy the conditions of the Frobenius theorem. However, we are eventually going to show that its integral manifolds are in correspondence with those of another - still invariant - Pfaffian system (a so-called prolongation) on $O^{\varepsilon}(k+n)$ which does satisfy the conditions of the Frobenius theorem. This implies that any connected integral submanifold of the prolonged Pfaffian system lies in a maximal such submanifold. We will see that these maximal submanifolds project to smooth 4-spheres in $G_{k}\left(\mathbf{R}^{k+n}\right)$ as mentioned in the Introduction; Theorem 1 then follows immediately.

So we begin analyzing the Pfaffian system

$$
\Phi=\operatorname{Span}\left\{\omega_{L}, \omega_{L R}, \omega_{2}, \omega_{3}, \omega_{4}\right\},
$$

where by $\omega_{L}, \& c$., we of course mean the various matrix-entry 1-forms. On any integral manifold $\hat{S} \subset O^{\varepsilon}(n+k)$ of $\Phi$ (that is, any manifold on which all forms in $\Phi$ vanish), the exterior derivatives of forms in $\Phi$ must vanish. Therefore, we consider these derivatives, computed using the Maurer-Cartan equation

$$
d\left(\begin{array}{cc}
\alpha & -{ }^{t} \omega \\
\omega & \beta
\end{array}\right)=-\left(\begin{array}{cc}
\alpha & -{ }^{t} \omega \\
\omega & \beta
\end{array}\right) \wedge\left(\begin{array}{cc}
\alpha & -{ }^{t} \omega \\
\omega & \beta
\end{array}\right)
$$

Dissected as above, we obtain (among others) the following equations:

$$
\begin{aligned}
& d \omega_{2} \equiv-\beta_{2} \wedge\left(\omega_{I}+\omega_{R}\right) \quad(\bmod \Phi), \\
& d \omega_{3} \equiv\left(\omega_{I}+\omega_{R}\right) \wedge{ }^{t} \alpha_{2} \quad(\bmod \Phi) \text {, } \\
& d \omega_{4} \equiv 0 \quad(\bmod \Phi) \text {. }
\end{aligned}
$$

The first two equations say that on an integral manifold of $\Phi$, we must have

$$
-\beta_{2} \wedge\left(\omega_{I}+\omega_{R}\right)=0, \quad\left(\omega_{I}+\omega_{R}\right) \wedge{ }^{t} \alpha_{2}=0 ;
$$

but a tedious application of the Cartan lemma shows that these latter equations, together with the independence condition $\omega^{1} \wedge \omega_{R}^{\mathbf{i}} \wedge \omega_{R}^{\mathbf{j}} \wedge \omega_{R}^{\mathbf{k}} \neq 0$, imply $\alpha_{2}=0$ and $\beta_{2}=0$, which we might have expected from Lemma 1. (Similarly, we expect to see conditions $\alpha_{L}=\beta_{L}$, coming shortly.) 
Now, it is clear from this last result that integral manifolds of $\Phi$ satisfying our independence condition are in one-to-one correspondence with integral manifolds of the partially prolonged Pfaffian system

$$
\Phi^{(1)}=\Phi \oplus \operatorname{Span}\left\{\alpha_{2}, \beta_{2}\right\}
$$

satisfying the same independence condition (where, once again, $\alpha_{2}$ and $\beta_{2}$ refer to the various 1 -form entries of those matrices). Now our situation is somewhat simpler, because in addition to the contrived

$$
d \omega_{2} \equiv 0, d \omega_{3} \equiv 0, d \omega_{4} \equiv 0 \quad\left(\bmod \Phi^{(1)}\right),
$$

we can also use (7) to compute

$$
d \alpha_{2} \equiv 0, d \beta_{2} \equiv 0 \quad\left(\bmod \Phi^{(1)}\right) .
$$

Therefore, all that remains to study are $d \omega_{L}$ and $d \omega_{L R}$. These are components of

$$
d \omega_{1} \equiv-\omega_{1} \wedge \alpha_{1}-\beta_{1} \wedge \omega_{1} \quad\left(\bmod \Phi^{(1)}\right) .
$$

We are also going to need derivatives of $\alpha_{1}$ and $\beta_{1}$ :

$$
d \alpha_{1} \equiv-\alpha_{1} \wedge \alpha_{1}+{ }^{t} \omega_{1} \wedge \omega_{1}, \quad d \beta_{1} \equiv \omega_{1} \wedge{ }^{t} \omega_{1}-\beta_{1} \wedge \beta_{1} \quad\left(\bmod \Phi^{(1)}\right) .
$$

Now observe that these equations for $d \omega_{1}, d \alpha_{1}, d \beta_{1}$ are identical to what one has in the special low-dimensional case $k=n=4$. No forms other than components of these $\omega_{1}, \alpha_{1}, \beta_{1}$ are going to make any further appearance, and we will therefore drop the subscript 1 when referring to them.

Let us then review what we have. There are skew-symmetric $4 \times 4$-matrices of 1 -forms $\alpha$ and $\beta$, decomposed as

$$
\alpha=\alpha_{L}^{\mu} L_{\mu}+\alpha_{R}^{\nu} R_{\nu}, \quad \beta=\beta_{L}^{\mu} L_{\mu}+\beta_{R}^{\nu} R_{\nu},
$$

and a $4 \times 4$-matrix of 1 -forms

$$
\omega=\omega^{1} I+\left(\omega_{L}^{\mu} L_{\mu}+\omega_{R}^{\nu} R_{\nu}\right)+\omega^{\mu \nu} L_{\mu} R_{\nu},
$$

satisfying

$$
\begin{aligned}
& d \omega \equiv-\omega \wedge \alpha-\beta \wedge \omega \quad\left(\bmod \Phi^{(1)}\right), \\
& d \alpha \equiv-\alpha \wedge \alpha+{ }^{t} \omega \wedge \omega \quad\left(\bmod \Phi^{(1)}\right), \\
& d \beta \equiv \omega \wedge{ }^{t} \omega-\beta \wedge \beta \quad\left(\bmod \Phi^{(1)}\right) .
\end{aligned}
$$

We are requiring $\omega_{L}^{\mu}$ and $\omega^{\mu \nu}$ to vanish, and $\omega^{1}, \omega_{R}^{\nu}, \alpha_{L}^{\mu}+\beta_{L}^{\mu}, \alpha_{R}^{\nu}$, and $\beta_{R}^{\nu}$ to be independent.

Differentiation of the equations $\omega_{L}^{\mu}=0$ and $\omega^{\mu \nu}=0$ will display more forms whose vanishing is required. For instance, the value of $d \omega_{L}^{\mathbf{i}}$ is the $L_{\mathbf{i}}$-component (referring to the decomposition (1) ) of the 2 -form matrix $d \omega$ given in (10). Remember that we only need this modulo $\Phi^{(1)}$ :

$$
\begin{aligned}
& d \omega_{L}^{\mathbf{i}} \equiv- {\left[\left(\omega^{1} I+\omega_{R}^{\nu} R_{\nu}\right) \wedge\left(\alpha_{L}^{\mu} L_{\mu}+\alpha_{R}^{\nu} R_{\nu}\right)\right.} \\
&\left.+\left(\beta_{L}^{\mu} L_{\mu}+\beta_{R}^{\nu} R_{\nu}\right) \wedge\left(\omega^{1} I+\omega_{R}^{\nu} R_{\nu}\right)\right]_{L}^{\mathbf{i}} \\
& \equiv\left(\alpha_{L}^{\mathbf{i}}-\beta_{L}^{\mathbf{i}}\right) \wedge \omega^{1} \quad\left(\bmod \Phi^{(1)}\right) .
\end{aligned}
$$

Similar computations give

$$
\begin{aligned}
d \omega_{L}^{\mu} & \equiv\left(\alpha_{L}^{\mu}-\beta_{L}^{\mu}\right) \wedge \omega^{1} \quad\left(\bmod \Phi^{(1)}\right), \\
d \omega^{\mu \nu} & \equiv\left(\alpha_{L}^{\mu}-\beta_{L}^{\mu}\right) \wedge \omega_{R}^{\nu} \quad\left(\bmod \Phi^{(1)}\right) .
\end{aligned}
$$


This shows that on any integral manifold of $\Phi^{(1)}$ satisfying the independence condition $\omega^{1} \wedge \omega^{\mathbf{i}} \wedge \omega^{\mathbf{j}} \wedge \omega^{\mathbf{k}} \neq 0$, one also has $\alpha_{L}^{\mu}-\beta_{L}^{\mu}=0$ (as expected from Lemma 1).

Consequently, we prolong again in a manner very similar to the preceding, and define

$$
\Phi^{(2)}=\Phi^{(1)} \oplus \operatorname{Span}\left\{\alpha_{L}^{\mu}-\beta_{L}^{\mu}\right\} .
$$

We know by construction that

$$
d \omega_{L}^{\mu} \equiv 0, \quad d \omega^{\mu \nu} \equiv 0 \quad\left(\bmod \Phi^{(2)}\right),
$$

and we finally compute from (11), 12):

$$
\begin{aligned}
d\left(\alpha_{L}^{\lambda}-\beta_{L}^{\lambda}\right) \equiv[ & -\left(\alpha_{L}^{\mu} L_{\mu}+\alpha_{R}^{\nu} R_{\nu}\right) \wedge\left(\alpha_{L}^{\mu} L_{\mu}+\alpha_{R}^{\nu} R_{\nu}\right) \\
& \left.+\quad\left(\omega^{1} I-\omega_{R}^{\nu} R_{\nu}\right) \wedge\left(\omega^{1} I+\omega_{R}^{\nu} R_{\nu}\right)\right]_{L}^{\lambda} \\
- & {\left[\left(\omega^{1} I+\omega_{R}^{\nu} R_{\nu}\right) \wedge\left(\omega^{1} I-\omega_{R}^{\nu} R_{\nu}\right)\right.} \\
& \left.-\left(\beta_{L}^{\mu} L_{\mu}+\beta_{R}^{\nu} R_{\nu}\right) \wedge\left(\beta_{L}^{\mu} L_{\mu}+\beta_{R}^{\nu} R_{\nu}\right)\right]_{L}^{\lambda} \\
\equiv[ & \left.-\alpha_{L}^{\mu} \wedge \alpha_{L}^{\nu} L_{\mu} L_{\nu}+\beta_{L}^{\mu} \wedge \beta_{L}^{\nu} L_{\mu} L_{\nu}\right]_{L}^{\lambda} \\
\equiv 0 & \left(\bmod \Phi^{(2)}\right) .
\end{aligned}
$$

Equations (8), (9), (13), and (14) show that the left-invariant Pfaffian system $\Phi^{(2)}$ on $O^{\varepsilon}(k+n)$ satisfies the conditions of the Frobenius theorem, and we already know that its integral manifolds are locally in one-to-one correspondence with $p_{1}$ calibrated submanifolds of $\mathcal{M}=G_{k}\left(\mathbf{R}^{k+n}\right)$.

We consider the maximal connected integral manifold of $\Phi^{(2)}$ passing through the identity $e \in S O(k+n, \mathbf{R}) \subset O^{\varepsilon}(k+n)$; let this submanifold be denoted by $\hat{S}$. The proof will be complete if we can show that this leaf projects to a smooth submanifold in $G_{k}\left(\mathbf{R}^{k+n}\right)$, as claimed above. To see this, note that the tangent space of $\hat{S}$ at any point, and in particular at the identity $e$, is defined as a subspace of the tangent space $\mathfrak{s o}(k+n, \mathbf{R})$ of $S O(k+n, \mathbf{R})$ by the equations

$$
\omega_{L}=0, \omega_{L R}=0, \omega_{2}=0, \omega_{3}=0, \omega_{4}=0, \alpha_{2}=0, \beta_{2}=0, \alpha_{L}-\beta_{L}=0 .
$$

But one can verify that at the identity, the tangent subspace defined by these equations is actually a Lie subalgebra of $\mathfrak{s o}(k+n, \mathbf{R})$. (Verifying closure of this space under the Lie bracket is essentially equivalent to verifying that the hypotheses of the Frobenius theorem are satisfied.) This, combined with left-invariance, tells us that our leaf $\hat{S}$ is a connected Lie subgroup of $S O(k+n, \mathbf{R})$, and that the other leaves are its cosets in $O^{\varepsilon}(k+n)$.

This subgroup may be described as follows, with reference to the decomposition (2) of $\mathbf{R}^{k+n}$. First note that the action of $\hat{S} \subset S O(k+n, \mathbf{R})$ preserves the subspaces $\mathbf{H} \oplus \mathbf{H}^{\prime}, \mathbf{R}^{k-4}$, and $\mathbf{R}^{n-4}$, and is arbitrary (but orthogonal) on the latter two components. On the component $\mathbf{H} \oplus \mathbf{H}^{\prime}$, our group includes the 10-dimensional group $S p(2)$ of linear Euclidean motions of $\mathbf{H}^{2}$ commuting with left-multiplication by (quaternion) scalars. (This corresponds to the non-zero forms $\alpha_{R}, \beta_{R}, \omega_{I}, \omega_{R}$.) $\hat{S}$ also includes that same left-multiplication by unit quaternions (which corresponds to the non-zero form $\alpha_{L}+\beta_{L}$ ). Consequently, the entire group $\hat{S}$ on $\mathbf{H} \oplus \mathbf{H}^{\prime}$ acts transitively on the collection of 1-dimensional quaternionic subspaces

$$
\left\{(x, y) \in \mathbf{H}^{2}: x a+y b=0\right\} \subset \mathbf{H} \oplus \mathbf{H}^{\prime} .
$$


The image under $\pi: O^{\varepsilon}(k+n) \rightarrow G_{k}\left(\mathbf{R}^{k+n}\right)$ of $\hat{S}$ is then equal to a quaternionic projective line $\mathbf{H P}^{1} \subset G_{4}\left(\mathbf{H} \oplus \mathbf{H}^{\prime}\right) \subset G_{k}\left(\mathbf{R}^{k+n}\right)$, with the canonical orientation (generated on tangent spaces by left-multiplication by $\mathbf{i}$, for instance, or derivable from the definition (5)).

This completes the proof of Theorem 1.

\section{The Grassmannian of Non-Oriented Subspaces}

At this point, it is easy to understand the $p_{1}$-calibrated submanifolds of the Grassmannian $G_{k}^{\natural}\left(\mathbf{R}^{k+n}\right)$ of non-oriented $k$-subspaces of $\mathbf{R}^{k+n}$. This space has the previously considered Grassmannian as a double-cover, and all of their respective local geometries are identical. It is crucial of course that $p_{1}$ is defined on $G_{k}^{\natural}\left(\mathbf{R}^{k+n}\right)$, which follows from its invariance under the orientation-reversal map $\varepsilon$ on $G_{k}\left(\mathbf{R}^{k+n}\right)$. The most apparent $p_{1}$-calibrated submanifold of $G_{k}^{\natural}\left(\mathbf{R}^{k+n}\right)$ is the set of 1-dimensional quaternionic subspaces under the identification (2), but without the information of the canonical quaternionic orientation. It is easy to verify that this is still a round 4 -sphere, which has as a double cover in $G_{k}\left(\mathbf{R}^{k+n}\right)$ the disjoint union of two spheres.

The main difference is that the symmetry group $O^{\varepsilon}(k+n)$ for $G_{k}\left(\mathbf{R}^{k+n}\right)$ is replaced by $O(k+n, \mathbf{R})$ acting on $G_{k}^{\natural}\left(\mathbf{R}^{k+n}\right)$ (that is, we take the quotient by the orientation-reversal map $\varepsilon$, which generates a normal subgroup of order 2 in $\left.O^{\varepsilon}(k+n)\right)$. The description of the $p_{1}$-calibrated planes is the same, except that only two components occur at each point of $G_{k}^{\natural}\left(\mathbf{R}^{k+n}\right)$ instead of four. Furthermore, the Lie algebra of the structure group is the same, and the invariance of the previously studied Pfaffian system with independence condition (on $\left.O^{\varepsilon}(k+n)\right)$ under $\varepsilon$ implies that there exists a Pfaffian system with independence condition on $O(k+n, \mathbf{R})$ which lifts to the former, and plays the same role. These two systems are locally isomorphic, and the analysis proceeds in exactly the same way, yielding the same "identity leaf" $\hat{S}$. Repeating our interpretation of the identical calculations then yields the following.

Theorem 2. Given any $p_{1}$-calibrated oriented 4-plane $V_{g} \subset T_{p} G_{k}^{\natural}\left(\mathbf{R}^{k+n}\right)$ there exists a unique maximal connected $p_{1}$-calibrated submanifold containing $p$ and tangent to $V_{g}$; any connected $p_{1}$-calibrated submanifold lies in a maximal one. These maximal submanifolds are round 4 -spheres.

Corollary 2. Any two maximal connected $p_{1}$-calibrated submanifolds of $G_{k}^{\natural}\left(\mathbf{R}^{k+n}\right)$ are congruent under the ambient isometry group.

\section{REFERENCES}

1. D. DeTurck, H. Gluck, C. Gordon, and D. Webb, You cannot hear the mass of a homology class, Comment. Math. Helv. 64 (1989), 589-617. MR 90k:58233

2. J. Dadok and R. Harvey, The Pontryagin 4-form, Proc. Amer. Math. Soc. 127 (1999), 31753180. MR 2000g:53058

3. H. Gluck, D. Mackenzie, and F. Morgan, Volume-minimizing cycles in Grassmann manifolds, Duke Math. J. 79 (1995), 335-404. MR 96d:53061

4. H. Gluck, F. Morgan, and W. Ziller, Calibrated geometries in Grassmann manifolds, Comment. Math. Helv. 64 (1989), 256-268. MR 90h:53077

5. M. Gromov, Carnot-Carathéodory spaces seen from within, Sub-Riemannian Geometry (A. Bellaiche and J. J. Risler, eds.), Progress in Mathematics, vol. 144, Birkhauser, 1996, pp. 79-323. MR 2000f:53034 
6. D. Grossman and W. Gu, Volume-minimizing cycles calibrated by the second Chern form. In preparation.

7. W. Gu, The stable 4-dimensional geometry of the real Grassmann manifolds, Duke Math. J. 93 (1998), 155-178. MR 99e:53091

8. R. Harvey and H. B. Lawson, Jr., Calibrated geometries, Acta Math. 148 (1982), 47-157. MR 85i:53058

9. C. Michael, Uniqueness of calibrated cycles using exterior diferential systems, Ph.D. thesis, Duke University, 1996.

10. J. Milnor and J. Stasheff, Characteristic Classes, Princeton University Press, Princeton, New Jersey, 1974. MR 55:13428

11. L.-H. Pan, Existence and uniqueness of volume-minimizing cycles in Grassmann manifolds, Ph.D. thesis, University of Pennsylvania, 1992.

12. G. Tian, Gauge theory and calibrated geometry, I, Ann. Math. 151 (2000), 193-268. MR 2000m:53074

13. J. A. Wolf, Spaces of Constant Curvature, McGraw-Hill, New York, 1967. MR 36:829

Department of Mathematics, Princeton University, Princeton, New Jersey 08544

Current address: Deparment of Mathematics, University of Chicago, 5734 University Avenue, Chicago, Illinois 60637

E-mail address: dan@math.uchicago.edu

Department of Mathematics, Harvey Mudd College, Claremont, California 91711

E-mail address: gu@math.hmc.edu 